\title{
Publikation von Urteilen durch Gerichte
}

\author{
«Urteile sind im Internet für die Öffentlichkeit zugänglich zu machen» ${ }^{1}$
}

Daniel Hürlimann*

«Ein Urteil, dessen Begründung die gebotene Überzeugungskraft hat, gewinnt weit über den Einzelfall hinaus Bedeutung, so dass es nicht nur die Parteien, sondern die Öffentlichkeit angeht.»²

Oskar Bosshardt, alt Präsident des Zürcher Verwaltungsgerichts

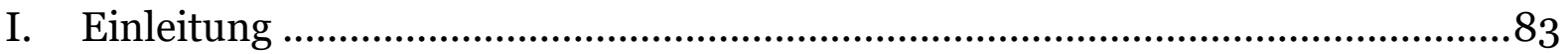

II. Aktuelle Publikationspraxis der Gerichte in der Schweiz .................................84

III. Rechtsgrundlagen für eine Publikationspflicht .................................................8 87

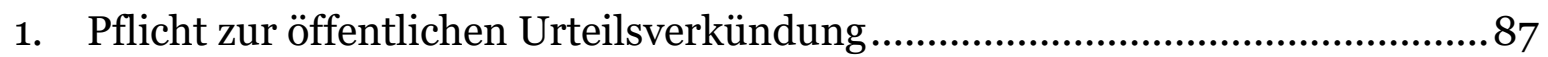

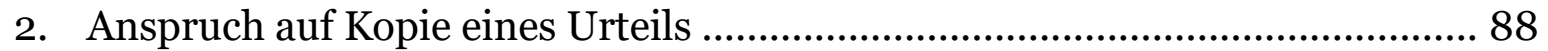

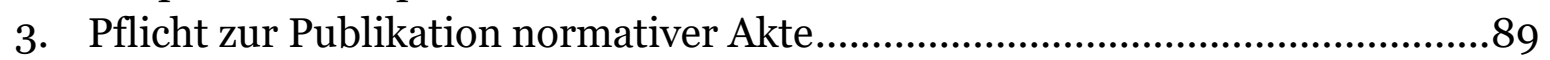

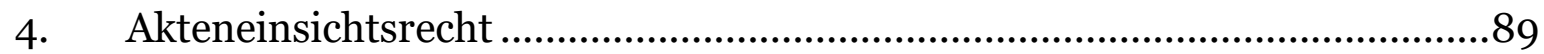

5. Öffentlichkeitsgesetze …………………………................................................90

6. Pflicht zur Zugänglichmachung gemäss ZPO...............................................92

IV. Umfang der Publikation ..................................................................................93

V. Argumente gegen eine umfassende Publikationspflicht ......................................95

1. Informationsüberflutung ............................................................................. 95

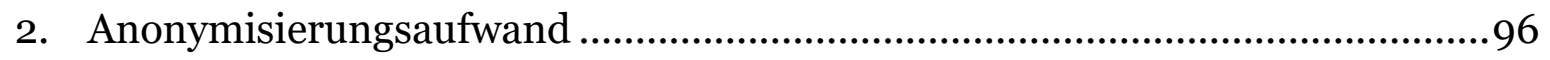

2.1. Aufwand der Anonymisierung....................................................................96

2.2. Notwendigkeit der Anonymisierung …………………................................96

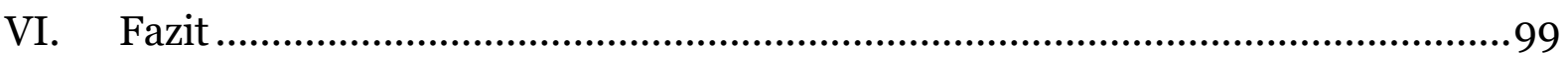

Zitiervorschlag: Daniel Hürlimann, Publikation von Urteilen durch Gerichte, in: suigeneris 2014, S. 82

URL: $\quad$ sui-generis.ch/8

DOI: $\quad$ https://doi.org/10.21257/sg.8

Dr. iur., Rechtsanwalt, CAS Judikative. Forschungsmitarbeiter an den Universitäten Luzern und Zürich. twitter.com/dhuerlimann.

Beim vorliegenden Text handelt es sich um eine überarbeitete Fassung der vom Autor im Rahmen der Richterakademie (CAS Judikative) im Herbst 2012 verfassten Abschlussarbeit. Weil es zu diesem Zeitpunkt noch keine Möglichkeit zur Open-Access-Publikation gab, erfolgt diese mit einer gewissen Verzögerung. Der Autor dankt Markus Felber, Thomas Stadelmann und Dominique Strebel für die kritische Durchsicht des Manuskripts. 


\section{Einleitung}

1 Anstoss zu dieser Arbeit gab Google: Obwohl es das erklärte Ziel dieses Unternehmens ist, «den Zugang zu Informationen weltweit in jeder Sprache zu ermöglichen"3, und obwohl Google selbst kommuniziert, dass im Jahr 2010 in der Schweiz acht «Court Orders" (gerichtliche Anordnungen) bezüglich «Content Removal» (Entfernung von Inhalten) eingegangen sind 4 , besteht keine Möglichkeit, an diese Urteile zu gelangen. Dies ist sowohl aus wissenschaftlicher als auch aus anwaltlicher Sicht und nicht zuletzt für Gerichte, die sich mit solchen Fällen zu befassen haben, unbefriedigend. Den endültigen Entschluss zum Verfassen dieser Arbeit habe ich nach erfolglosen Anfragen um Zustellung von Urteilen bei Gerichten in den Kantonen Basel-Stadt5, Bern 6 , Thurgau ${ }^{7}$ und Zug 8 gefasst.

2 Während Beschlüsse der Exekutive und Legislative sowohl auf eigednössicher als auch auf kantonaler Ebene allgemein zugänglich sind, gilt dies nur beschränkt für Gerichtsurteile. Die Gründe dafür sind, abgesehen vom Aufwand, der eine Publikation mit sich bringt, nicht auf den ersten Blick ersichtlich - im Gegenteil: Schutzzwecke der Urteilsöffentlichkeit sind die Unterbindung von Geheimjustiz, die Transparenz der richterlichen Erkenntnisse und die Unterrichtung der Öffentlichkeit über die Rechtspraxis9. Es ist jedoch fraglich, ob diesen Schutzzwecken mit dem Wortlaut der Verfassung Genüge getan wird, wenn diese in Art. 30 Abs. 3 BV nur die Öffentlichkeit der Urteilsverkündung verlangt.

3 Das Bundesgericht hat die Bedeutung der Justizöffentlichkeit und der daraus abgeleiteten Informationsrechte folgendermassen konkretisiert: «Sie sorgen für Transparenz in der Rechtspflege, was eine demokratische Kontrolle durch das Volk erst ermöglicht, und bedeuten damit eine
1 Hans Wiprächtiger, Öffentlichkeit und Justiz, in: Bernhard Ehrenzeller / Urs Saxer (Hrsg.), St. Galler Tagung zur Öffentlichkeitskommunikation des Staates, St. Gallen 2010, S. 145 ff., S. 147.

2 Oskar Bosshardt, Bemerkungen zur Veröffentlichung von Entscheiden, ZB1 67/1966, S. 345.

3 www.google.com/intl/de/about/company/philosophy.

4 www.google.com/transparencyreport/removals/government/CH/?p=2010-06 (4 gerichtliche Verfügungen von Januar bis Juni 2010) bzw. www.google.com/transparencyreport/removals/government/CH/?p=2010-12 (4 gerichtliche Verfügungen von Juli bis Dezember 2010).

5 Schreiben des Strafgerichts Basel-Stadt vom 2. April 2012: «Das Urteil i.S. [Name] ist bisher ausschliesslich mündlich begründet worden. Falls ein schriftliches Urteil erstellt würde, wird es ausschliesslich den Parteien gestellt [sic] und kann nicht von Drittpersonen angefordert werden. Ihrem Gesuch um Zustellung eines Urteils wird daher nicht entsprochen.»

6 Telefonische Auskuft eines Gerichtsschreibers des
Regionalgerichts Oberland in einem Strafprozess, bei dem der Autor dieses Textes als Zeuge aussagen musste.

7 E-Mail des Obergerichts des Kantons Thurgau vom 16. April 2012: «Bitte senden Sie und ein schriftliches Gesuch mit der Begründung, weshalb Sie eine Kopie dieses Entscheids benötigen.»

8 E-Mail des Kantonsgerichts Zug vom 23. Mai 2011: «Darf ich Sie zunächst anfragen, wie Sie Kenntnisnahme des betreffenden Urteils erlangt haben? Was die Herausgabe von Urteilen anbelangt, verweist das Kantonsgericht Dritte zunächst grundsätzlich an die Verfahrensparteien. Vorliegend könnte das Urteil lediglich in anonymisierter Form herausgegeben werden, was mit Kosten von rund CHF 50.- für den Aufwand verbunden wäre.»

9 Niccolò Raselli, Das Gebot der öffentlichen Urteilsverkündung, in: Dietmar Mieth/René Pahud de Mortanges (Hrsg.), Recht - Ethik - Religion, Der Spannungsbogen für aktuelle Fragen, historische Vorgaben und bleibende Probleme, Luzern 2002, S. 23 ff., S. 24. 
Absage an jede Form geheimer Kabinettsjustiz. Ohne Gerichtsöffentlichkeit sind Spekulationen, ob die Justiz einzelne Prozessparteien ungebührlich benachteiligt oder privilegiert, unvermeidlich. Kritik an einseitiger oder rechtsstaatlich fragwürdiger Ermittlungstätigkeit oder mangelhafter Verfahrensleitung bliebe ausgeschlossen ${ }^{10}$. Das Bundesgericht hat mit seiner Rechtsprechung zu Art. 30 Abs. 3 BV, der nur Gerichtsverhandlung und Urteilsverkündung für öffentlich erklärt, den Geltungsbereich dieser Bestimmung somit ausgedehnt ${ }^{11}$.

4 Die vorliegende Arbeit vermittelt $\mathrm{zu}$ nächst den Status quo der Urteilspublikation auf Bundesebene und in den Kantonen, bevor untersucht wird, ob und in welchem Umfang Urteile publiziert werden müss(t)en und was allenfalls dagegen sprechen könnte. Ein nicht selten gegen die Publikation von Urteilen vorgebrachtes Argument ist der Anonymisierungsaufwand. In diesem Zusammenhang ist im Rahmen der vorliegenden Arbeit auch zu prüfen, ob eine durchgängige Anonymisierung nötig und sinnvoll ist, wobei auch der Aufwand für die Anonymisierung von Urteilen berücksichtigt werden soll.

\section{Aktuelle Publikationspraxis der Ge- richte in der Schweiz}

5 Die vier Gerichte des Bundes publizieren grundsätzlich all ihre Urteile. Seit der vom Volk im Jahr 2000 angenommenen ${ }^{12}$ Justizreform ist die Information der Öffentlichkeit über die Rechtsprechung im Bundesgerichtsgesetz (Art. 27 BGG $^{13}$ ), im Verwaltungsgerichtsgesetz (Art. $29 \mathrm{VGG}^{14}$ ) und im Strafbehördenorganisationsgesetz (Art. 63 StBOG ${ }^{15}$ ) festgehalten; ebenso wird sie im Patentgerichtsgesetz verlangt (Art. 25 PatGG $^{16}$ ).

6 Das Bundesgericht veröffentlicht seit $1875^{17}$ eine Auswahl von Leitentscheiden in der amtlichen Sammlung (BGE ${ }^{18}$ ) und ist im Jahr 2000 dazu übergegangen, zusätzlich ungefähr die Hälfte aller Urteile ${ }^{19}$ und ab 2007 sämtliche End- und Teilentscheide sowie die vom Abteilungspräsidium bezeichneten Vor- und Zwischenentscheide in anonymisierter Form im Internet aufzuschalten ${ }^{20}$.

7 Bundesverwaltungsgericht und Bundesstrafgericht veröffentlichen seit der Aufnahme ihrer Tätigkeit alle Urteile online21, ebenfalls durchgängig anonymisiert. Vergleichbar mit den BGE bringen
10 BGE 137 I 16 E. 2.2 S. 19.

11 Dominique Strebel, Justizdokumente, in: Catherine Boss / Dominique Strebel, Recherche in der Praxis, Zürich 2012, S. 108-132, S. 110.

12 Volksabstimmung vom 12.03.2000.

13 Bundesgesetz vom 17. Juni 2005 über das Bundesgericht (Bundesgerichtsgesetz, BGG, SR 173.110).

14 Bundesgesetz vom 17. Juni 2005 über das Bundesverwaltungsgericht (Verwaltungsgerichtsgesetz, VGG, 173.32).

15 Bundesgesetz vom 19. März 2010 über die Organisation der Strafbehörden des Bundes (Strafbehördenorganisationsgesetz, StBOG, SR 173.71).

16 Bundesgesetz vom 20. März 2009 über das Bundespatentgericht (Patentgerichtsgesetz, PatGG, 173.41).
17 Paul Tschümperlin, Die Publikation gerichtlicher Entscheide, in: Daniel Kettiger / Thomas Sägesser (Hrsg.), Kommentar zum Publikationsgesetz des Bundes, Bern 2011, S. 69 ff., N 8.

18 Vgl. auch Art. 58 Abs. 1 des Reglements für das Bundesgericht (BGerR): Entscheide von grundsätzlicher Bedeutung werden in der Amtlichen Sammlung veröffentlicht.

19 Heinz Aemisegger, Öffentlichkeit der Justiz, in: Pierre Tschannen (Hrsg.), Neue Bundesrechtspflege, Bern 2007, S. 375 ff., S. 400.

20 Art. 59 Abs. 2 lit. b BGerR.

21 Vgl. Art. 5 des Informationsreglements für das Bundesverwaltungsgericht bzw. Art. 3 Abs. 1 des Reglements des Bundesstrafgerichts über die Grundsätze der Information. 
auch das Bundesverwaltungsgericht und das Bundesstrafgericht eine amtliche Sammlung heraus ${ }^{22}$ : die Entscheide des schweizerischen Bundesverwaltungsgerichts (BVGE) bzw. die Entscheide des schweizerischen Bundesstrafgerichts (TPF).

8 Auch das Bundespatentgericht als - im wahrsten Sinne des Wortes - jüngstes Gericht veröffentlichte von Anbeginn, d.h. seit dem Jahr 2012, sämtliche Entscheide auf seiner Webseite. Im Unterschied zu den anderen Gerichten des Bundes wird hier aber von einer Anonymisierung im Grundsatz abgesehen ${ }^{23}$ und es existiert auch keine amtliche Sammlung von Entscheiden des Bundespatentgerichts 24 .

9 Weit weniger einheitlich wird die Publikation von Gerichtsurteilen auf kantonaler Ebene gehandhabt, wie eine in sämtlichen Kantonen durchgeführte Umfrage (Stand 201225) zeigt. Mit Ausnahme des Kantons Glarus ${ }^{26}$ publizierten im Jahr 2012 zwar alle Kantone Urteile, kein Kanton verfolgt aber dabei den Grundsatz, dass sowohl inhaltlich als auch bezogen auf die verschiedenen Gerichte und Instanzen sämtliche Entscheide zu publizieren sind. Insgesamt 6 Kantone (BL, GR, LU, TI, VD, ZH) veröffentlichen allerdings schon alle Sachurteile des Kantons-/Obergerichts bzw. der oberen kantonalen Gerichte. Alle anderen Kantone nehmen eine inhaltliche Auswahl vor, wobei die meisten Kantone (Ausnahmen: AI, BS, GE, VS, ZG) zusätzlich eine Beschränkung auf die oberen kantonalen Gerichte kennen.

10 In neun Kantonen bestehen Pläne zum Ausbau der Publikation von Urteilen: Im Kanton Basel-Stadt war ursprünglich für 2013 die Einführung einer eigenen elektronischen Publikation der Urteile des Appellationsgerichts geplant. Im Kanton Bern ist seit 2012 eine Webpublikation mit besseren Suchmöglichkeiten geplant. Im Kanton Freiburg sieht das am 1. September 2012 in Kraft getretene Reglement über die Information der Öffentlichkeit in Gerichtssachen ${ }^{27}$ eine vermehrte Publikation von Gerichtsurteilen vor. Im Kanton Genf gibt es zusätzliche personelle Ressourcen für die Anonymisierung und im Kanton Glarus werden seit Kurzem einzelne Urteile des Obergerichts (Zivil- und Strafsachen) und des Verwaltungsgerichts auf dem Internet publiziert. Der Kanton Zug prüfte im Jahr 2012, ob die jährlich als Gerichts- und Verwaltungspraxis (GVP) publizierten Entscheide in Zukunft fortlaufend, d.h. nicht mehr erst im folgenden Jahr, publiziert werden sollen und hat sich offenbar dagegen entschieden oder den Entscheid bisher nicht umgesetzt ${ }^{28}$. Schliesslich war auch in den
22 Art. 7 des Informationsreglements für das Bundesverwaltungsgericht bzw. Art. 5 des Reglements des Bundesstrafgerichts über die Grundsätze der Information.

23 Art. 3 Abs. 3 des Informationsreglements für das Bundespatentgericht vom 28. September 2011 (IRPatGer): «Die Veröffentlichung erfolgt in nicht anonymisierter Form, es sei denn, der Schutz privater oder öffentlicher Interessen erfordert eine Anonymisierung. Diese kann von Amtes wegen vorgenommen werden. Bei privaten Interessen wird die Anonymisierung vorgenommen, wenn sie beantragt wird und begründet erscheint.»

24 Gemäss Art. 3 Abs. 2 IR-PatGer werden jedoch wichtigen Entscheiden Regesten in den drei Amtssprachen vorangestellt.

25 Vgl. die Übersicht der Antworten, abrufbar unter http://tinyurl.com/urteilspub.

26 Inzwischen existiert auch im Kanton Glarus eine Entscheiddatenbank der Gerichte.

27 Reglement des Kantonsgerichts vom 21. Juni 2012 über die Information der Öffentlichkeit in Gerichtssachen (InfoRKG, ASF 2012_080).

28 Auf der Webseite des Kantons Zug zur Gerichts- 
Kantonen Genf, Luzern, Nidwalden und Wallis ein Ausbau geplant, wobei im Zeitpunkt der Umfrage noch keine konkreten Pläne vorlagen.

11 In den meisten Kantonen werden sämtliche Urteile anonymisiert. Ausnahmen kennen die Kantone Appenzell Innerrhoden (wenn eine Streitsache bereits öffentlich bekannt ist), Genf (Anonymisierung nur bei «décisions sensibles» ${ }^{29}$ ), Luzern (i.d.R. keine Anonymisierung im Medienraum ${ }^{30}$ ), Schaffhausen (keine Anonymisierung bei fehlendem Schutzinteresse ${ }^{31}$ ), Waadt (Anonymisierung abhängig vom Rechtsgebiet32) und Zürich (allenfalls keine Anonymisierung bei namens- und markenrechtlichen Angelegenheiten).

Mit Ausnahme des Kantons Thurgau können die Urteile in allen Kantonen beim Gericht vor Ort eingesehen werden. In 13 Kantonen 33 können die vollständigen Urteile eingesehen werden, in 8 Kantonen 34 nur das Dispositiv35. Meistens ist diese Einsichtsmöglichkeit zeitlich beschränkt: die Dauer bewegt sich zwischen 3 Tagen im Kanton Zug und 3 Monaten im Kanton Basel-Stadt, verbreitet sind 30 Tage ${ }^{6}$. 7 Kantone kennen keine zeitliche Beschränkung37.

13 In allen Kantonen ausser Appenzell Innerrhoden, Obwalden, Thurgau und Uri können Urteile beim Gericht bestellt werden. Die Kantone Freiburg, Solothurn, Wallis und Zug schränken diese Möglichkeit auf begründete Fälle wie z.B. Geltendmachung eines wissenschaftlichen Interesses ein. Einem Gesuch um Zustellung aller Urteile aus einem bestimmten Rechtsgebiet (z.B. Immaterialgüterrecht) würde in 9 Kantonen entsprochen ${ }^{38}$, in 13 Kantonen würde es dagegen abgelehnt39 und 4 Kantone behalten sich eine Einzalfallprüfung vor40. 10 Kantone gaben an, ihre Publikationspraxis durch das Inkrafttreten der ZPO angepasst bzw. ausgebaut zu haben ${ }^{41}$. und Verwaltungspraxis ist das aktuellste Dokument die Gerichts- und Verwaltungspraxis 2013.

29 P.ex. cf. protection de la sphère privée ou intérêt digne de protection, selon l'appréciation de la juridiction concernée.

3o Hier wird nur im Einzelfall anonymisiert; die Regel ist keine Anonymisierung (z.B. aber ganz oder teilweise Anonymisierung zum Schutz von Kindern, Opfern sexueller Gewalt). Anzufügen gilt, dass im Medienraum z.B. noch nie ein Scheidungsurteil veröffentlicht worden ist. Es müssen schon Fälle sein, die für die Medien attraktiv, vermittelbar und (inhaltlich) von öffentlichem Interesse sind.

${ }^{31}$ Z.B. Behörden, Gemeinden, Heimatschutz.

32 Certains arrêts de la Cour de droit administratif et public ne sont pas anonymisés. C'est le cas s'agissant des décisions concernant l'aménagement du territoire et des constructions, les améliorations foncières, les estimations fiscales et les taxes communales. Il est possible que de cas en cas certaines autres décisions de ladite cour ne soient pas anonymisées.
33 AR, AI, BS, FR, GE, JU, LU, NE, OW, SH, SO, UR, ZG.

34 AG (nur nicht öffentlich verkündete Endentscheide), BL, BE, NW, SZ, SG, VS, ZH.

35 In den Kantonen Glarus und Waadt wurde die Einsicht beim Gericht gemäss Auskunft der befragten GerichtsmitarbeiterInnen noch nie verlangt. Von den Kantonen Graubünden und Tessin wurde die Frage nicht beantwortet.

36 AG, BL, FR, LU, NW, OW, SH, SZ, SO, ZH; BE: 15 Arbeitstage; SG: 14 Tage.

37 AR, GE, JU, NE, UR, VD, VS

38 AR, BS (nur bei klarer Begrenzung, d.h. weniger als 10 Urteile), GE, NE; nur für wissenschaftliche Zwecke: BL, GL, NW, SH, VD.

39 AI, BE (bei wissenschaftlichen Zwecken jedoch denkbar), FR, GR, OW, SZ, SO, TI, TG, UR, VS, $\mathrm{ZG}, \mathrm{ZH}$.

40 AG, JU, LU, SG.

41 AI, BL, BE, FR, GL, JU, NE, SH, SZ, ZH. 


\section{Rechtsgrundlagen für eine Publika- tionspflicht}

14 Nachfolgend wird untersucht, ob eine allgemeine Publikationspflicht in Bezug auf Urteile besteht bzw. auf welche Rechtsgrundlagen sich eine solche allenfalls stützt. Nebst der verfassungs- und völkerrechtlich festgeschriebenen Pflicht zur öffentlichen Urteilsverkündung werden die Pflicht zur Publikation normativer Akte, das Akteneinsichtsrecht sowie die Öffentlichkeitsgesetze herangezogen, bevor abschliessend ein Blick auf Art. 54 Abs. 1 Satz 2 der Zivilprozessordnung geworfen wird. Dieser lautet: «Die Entscheide werden der Öffentlichkeit zugänglich gemacht.»

\section{Pflicht zur öffentlichen Urteilsver- kündung}

15 Im Kapitel Grundrechte verlangt die Verfassung die Öffentlichkeit von Gerichtsverhandlung und Urteilsverkündung (Art. 30 Abs. 3 BV). Die Gerichtsöffentlichkeit hat sowohl eine rechtsstaatliche als auch eine demokratische Funktion: In ihrer rechtsstaatlichen Komponente dient die Transparenz der fairen Behandlung der Prozessbeteiligten, in ihrer demokratischen Funktion erlaubt sie es der Bevölkerung, sich an konkreten Beispielfällen über die Rechtsdurchsetzung zu informieren und über die wirksame und angemessene Erfüllung der Richteraufgabe zu wachen 42 .

16 Aus dem Wortlaut von Art. 30 Abs. 3 BV lässt sich kein direkter Anspruch auf Öffentlichkeit des Urteilstextes ableiten, da nur die Verkündung des Urteils genannt ist. Übereinstimmend besagt Art. 6 Abs. 1 EMRK, dass das Urteil öffentlich verkündet werden muss 43 und gleiche Garantien enthält auch Art. 14 Ziff. 1 UNO-Pakt II44. Eine aus BV, EMRK oder UNO-Pakt II direkt abgeleitete Pflicht zur Verkündung der Urteile durch Publikation besteht somit nicht 45 .
42 Franz Zeller, Gerichtsöffentlichkeit als Quelle der Medienberichterstattung, medialex 2003, S. 15 ff., S. 16.

43 Art. 6 Abs. 1 EMRK: «Jede Person hat ein Recht darauf, dass über Streitigkeiten in Bezug auf ihre zivilrechtlichen Ansprüche und Verpflichtungen oder über eine gegen sie erhobene strafrechtliche Anklage von einem unabhängigen und unparteiischen, auf Gesetz beruhenden Gericht in einem fairen Verfahren, öffentlich und innerhalb angemessener Frist verhandelt wird. Das Urteil muss öffentlich verkündet werden; Presse und Öffentlichkeit können jedoch während des ganzen oder eines Teiles des Verfahrens ausgeschlossen werden, wenn dies im Interesse der Moral, der öffentlichen Ordnung oder der nationalen Sicherheit in einer demokratischen Gesellschaft liegt, wenn die Interessen von Jugendlichen oder der Schutz des Privatlebens der Prozessparteien es verlangen oder - soweit das Gericht es für unbedingt erforderlich hält - wenn unter besonderen Umständen eine öffentliche Verhandlung die Interessen der Rechtspflege beeinträchtigen würde.» (Hervorhebungen hinzugefügt). vor Gericht gleich. Jedermann hat Anspruch darauf, dass über eine gegen ihn erhobene strafrechtliche Anklage oder seine zivilrechtlichen Ansprüche und Verpflichtungen durch ein zuständiges, unabhängiges, unparteiisches und auf Gesetz beruhendes Gericht in billiger Weise und öfentlich verhandelt wird. Aus Gründen der Sittlichkeit, der öffentlichen Ordnung (ordre public) oder der nationalen Sicherheit in einer demokratischen Gesellschaft oder wenn es im Interesse des Privatlebens der Parteien erforderlich ist oder - soweit dies nach Auffassung des Gerichts unbedingt erforderlich ist - unter besonderen Umständen, in denen die Öffentlichkeit des Verfahrens die Interessen der Gerechtigkeit beeinträchtigen würde, können Presse und Öffentlichkeit während der ganzen oder eines Teils der Verhandlung ausgeschlossen werden; jedes Urteil in einer Straf-oder Zivilsache ist jedoch öffentlich zu verkünden, sofern nicht die Interessen Jugendlicher dem entgegenstehen oder das Verfahren Ehestreitigkeiten oder die Vormundschaft über Kinder betrifft.» (Hervorhebungen hinzugefügt).

45 Caroline Flühmann / Patrick Sutter, Kritische Be- 
Mit Blick auf Sinn und Zweck der verfassungs- und völkerrechtlich garantierten Urteilsöffentlichkeit ist es jedoch fraglich, ob eine einmalige Verlesung oder die zeitlich beschränkte Auflage im Gerichtsgebäude genügen. Schutzzwecke der Urteilsöffentlichkeit sind die Unterbindung von Geheimjustiz, die Transparenz der Justiztätigkeit und die Schaffung von Vertrauen in den Rechtsstaat46. Während bei einer öffentlichen Urteilsverkündung von Geheimjustiz nicht die Rede sein kann, wird das Vertrauen in den Rechtsstaat durch die Publikation eines Urteils zweifellos zusätzlich gestärkt. Entscheidend ist aber m.E. die Transparenz der Justiztätigkeit, welche mit einer einmaligen öffentlichen Verkündung nur ansatzweise erreicht wird und erst bei einer Urteilspublikation vollumfänglich gegeben ist.

Die Schutzzwecke der Urteilsöffentlichkeit sind insbesondere auch nicht etwa abhängig von der Instanz, welche das in Frage stehende Urteil gefällt hat47. Die Veröffentlichung von Urteilen ist nicht zuletzt auch Basis für die wissenschaftliche Auseinandersetzung mit der Gerichtspraxis, was wiederum für die Weiterentwicklung von Rechtsprechung und $\mathrm{Ge}$ setzgebung grundlegend ist48. Das Bundesgericht hat bereits im Jahr 2001 festgehalten, dass dem Zweck des Verkündungsgebots mit einer Publikation in Pe- riodika oder auf dem Internet besser gedient sein dürfte als mit einer mündlichen Eröffnung des Urteils an einer Gerichtsverhandlung, da das Publikum faktisch nur begrenzte Möglichkeiten hat, an derartigen Verhandlungen teilzunehmen 49.

19 Als Zwischenergebnis kann festgehalten werden, dass bereits die Pflicht zur öffentlichen Urteilsverkündung gemäss Verfassung, EMRK und UNO-Pakt II mit einer Urteilspublikation ungleich besser erfüllt wird als mit dem einmaligen Verlesen im Gerichtssaal ggf. kombiniert mit einer öffentlichen Auflage.

\section{Anspruch auf Kopie eines Urteils}

20 In einem Urteil aus dem Jahr 2006 (nicht als BGE publiziert) hat das Bundesgericht festgehalten, dass dem Anspruch auf öffentliche Urteilsverkündung dann Genüge getan wird, "wenn das Urteil bei einer der Öffentlichkeit zugänglichen Kanzlei aufgelegt wird, wo jedermann, der ein berechtigtes Interesse glaubhaft machen kann, den vollständigen Text des Urteils einsehen oder sich gegen eine allfällige Gebühr eine Kopie erstellen lassen kann; weitergehende Ansprüche - insbesondere auf Zustellung einer Kopie - bestehen dagegen, gestützt auf die genannten Bestimmungen, nicht»50. trachtung der bundesgerichtlichen Veröffentlichungspraxis oder «Wünschbares ist machbar», AJP 2003, S. 1026 ff., S. 1035.

46 Gerold Steinmann, in: Bernhard Ehrenzeller / Benjamin Schindler / Rainer J. Schweizer / Klaus A. Vallender (Hrsg.), Die Schweizerische Bundesverfassung, St.Galler Kommentar, 3. Auflage, Zürich/St. Gallen 2014, Art. 30 N 43; Wiprächtiger (Fn. 1), S. 146 ff.

47 Aemisegger (Fn. 19), S. 389.

48 Bosshardt (Fn. 2), S. 346; Stefan Vogel, in: Isabelle
Häner / Markus Rüssli / Evi Schwarzenbach (Hrsg.), Kommentar zur Zürcher Kantonsverfassung, Zürich 2007, Art. 78 N 32.

49 Urteil des Bundesgerichts 1P.229/2001 vom 2. Oktober 2001, E. 2c.

50 Urteil des Bundesgerichts 1P.298/2006 vom 1. September 2006, E. 2.2 (Hervorhebung hinzugefügt), mit Verweis auf BGE 124 IV 234, aus dem jedoch (noch) nicht klar hervorging, ob sich die Aussage nur auf Strafurteile beziehen sollte. 
3. Pflicht zur Publikation normativer Akte

21 Die Pflicht zur Publikation normativer Akte ist in der Verfassung nicht explizit erwähnt ${ }^{51}$, ergibt sich aber aus dem Rechtsstaatsprinzip (Art. 5 BV) und aus der Pflicht zur Wahrung von Treu und Glauben (Art. $9 \mathrm{BV}$ ) ${ }^{2}$.

Im Rahmen der Globalisierung verschwinden zunehmend die Unterschiede zwischen dem präjudizienorientierten Common Law und dem ursprünglich einzig dem Gesetz verpflichteten Civil Law53. Auch in der Schweiz werden das Fallrecht und die Kenntnis desselben daher immer wichtiger54. Dies wiederum setzt voraus, dass das massgebende Fallrecht für jedermann zugänglich ist. Damit stellt sich die Frage, ob eine Unterscheidung in massgebendes und nicht massgebendes Fallrecht sinnvoll bzw. überhaupt möglich ist. Auf diese Frage wird im vierten Teil dieser Arbeit (Umfang der Publikationspflicht) eingegangen.

Die Publikation von Urteilen aufgrund ihres Präjudizcharakters ist auch für die Chancengleichheit im Prozess zentral, weil es Behörden und Richterinnen (die gleichzeitig auch als Anwältinnen tätig sein können55) insbesondere durch interne Datenbanken leichter fällt, sich über die Entwicklung der Rechtsprechung auf dem Laufenden zu halten 56 .

\section{Akteneinsichtsrecht}

24 Das Akteneinsichtsrecht ist Bestandteil des Anspruchs auf rechtliches Gehör gemäss Art. 29 Abs. 2 BV57. Ein Gericht, das sich auf einen früheren, aber nicht publizierten Entscheid abstützt, verletzt elementare Aspekte der Fairness, wie sie die EMRK, der UNO-Pakt II und die BV garantieren58. Aufgrund der verbreiteten Copy-Paste-Technik im Rahmen der Begründung von Gerichtsentscheiden besteht darüber hinaus die Gefahr, dass eine bereits früher verwendete Argumentation übernommen wird, ohne dass dies für die Parteien ersichtlich ist.

25 Man sollte meinen, dass sich ein Gericht davor hütet, explizit auf einen früher gefällten, aber nicht publizierten Entscheid abzustellen. Eine Untersuchung von Entscheiden des Bundesgerichts aus der Zeit vor der Veröffentlichung aller Urteile hat allerdings ganz andere Ergebnisse zutage gefördert: So wurden z.B. in BGE 96 II 69 insgesamt 22 Präjudizien zitiert, wovon 11 amtlich unveröffentlichte Bundesgerichtsentscheide waren; in BGE $103 \mathrm{~V} 177$ waren 8 von 14 zitierten Entscheiden
51 Vgl. auch Ingress des Publikationsgesetzes, demgemäss sich die Bundesversammlung beim Erlass desselben auf Art. 173 Abs. 2 BV (Zuständigkeit der Bundesversammlung für Geschäfte, die in die $\mathrm{Zu}$ ständigkeit des Bundes fallen und keiner anderen Behörde zugewiesen sind) gestützt hat.

52 Flühmann/Sutter (Fn. 45), S. 1032.

53 Vogel (Fn. 48), Art. 78 N 9; Pascal Pichonnaz, / Pierre Scyboz, Les dissenting opinions dans les jugements: une innovation à craindre?, SJZ 98/2002, S. 377 ff., S. 380.

54 Flühmann/Sutter (Fn. 45), S. 1032; Georg Müller /
Marc Thommen, Unabhängigkeit versus Öffentlichkeit der Justiz, in: Marianne Heer/Adrian Urwyler (Hrsg.), Justiz und Öffentlichkeit - Justice et public, Bern 2007, S. 23 ff., S. 25.

55 Regina Kiener / Gabriela Medici, Anwälte und andere Richter, SJZ 2011, S. 373 ff.

56 Vogel (Fn. 48), Art. 78 N 10.

57 Reinhold Hotz, in: Bernhard Ehrenzeller / Philippe Mastronardi / Rainer J. Schweizer / Klaus A. Vallender (Hrsg.), Die schweizerische Bundesverfassung, Kommentar, 2. Auflage, Zürich 2008, Art. 29 N 24; BGE 126 I 7 E. $2 b$.

${ }_{58}$ Flühmann/Sutter (Fn. 45), S. 1038. 
nicht publiziert59. Es kommt auch vor, dass ein unpublizierter Entscheid einzig mit dem Datum zitiert wird, sodass ihn das entscheidfällende Gericht selbst auf Gesuch hin nicht mehr findet ${ }^{60}$. In einem anderen Fall hat die amtlich publizierte Rechtsprechung während 18 Jahren nicht der effektiven Rechtsprechung entsprochen, obwohl in dieser Zeit verschiedene Urteile zu dieser Rechtsfrage ergingen ${ }^{61}$.

26 Diese Fälle zeigen, dass das Akteneinsichtsrecht letztlich nur dann vollumfänglich gewährt ist, wenn die Parteien bzw. ihre Vertreter die gleichen Möglichkeiten zur Einsicht und Recherche in den Urteilen eines Gerichts haben, wie dieses selbst. Dies ist zwar nicht einzig durch eine vollumfängliche Veröffentlichung der Rechtsprechung möglich. Der Aufbau einer umfassenden und durchsuchbaren Datenbank nur für Parteien und deren Vertreter erscheint indessen kaum sinnvoll bzw. es ist kein Grund ersichtlich, weshalb eine solche Datenbank nicht öffentlich zugänglich sein sollte.

\section{5. Öffentlichkeitsgesetze}

27 Das Öffentlichkeitsgesetz des Bundes heisst mit vollem Namen «Bundesgesetz über das Öffentlichkeitsprinzip der Verwaltung», was bereits daran zweifeln lässt, ob die Justiz in dessen Anwendungsbereich fällt. Art. 3 BGÖ (Sachlicher Geltungsbereich) hält denn auch fest, dass das Gesetz für den Zugang zu amtlichen Dokumenten betreffend Zivil- und Straf- sowie Verfahren der Staats- und Verwaltungsrechtspflege nicht gilt ${ }^{62}$. Entgegen den Ausführungen in der Botschaft, wonach Art. 3 BGÖ sowohl die hängigen als auch die abgeschlossenen Verfahren erfasst $^{63}$, wird mit überzeugenden Argumenten dafür gehalten, dass sich die Bestimmung nur auf hängige Verfahren beziehen kann64. Im Rahmen der vorliegenden Arbeit spielt diese Streitfrage jedoch nur für ältere Urteile eine Rolle, weil die Gerichte auf Bundesebene ihre jüngeren Entscheide bereits gestützt auf die entsprechenden Prozessgesetze veröffentlichen.

28 Die meisten Kantone ${ }^{65}$ (Ausnahmen: AI, GL, GR66, LU67, NW, OW und TG) haben ebenfalls ein Öffentlichkeits- oder Informationsgesetz bzw. entsprechende Verfassungsbestimmungen erlassen, wobei diese in der Mehrheit (Ausnahmen68: AG,
59 Thomas Probst, Die Änderung der Rechtsprechung, Eine rechtsvergleichende, methodologische Untersuchung zum Phänomen der höchstrichterlichen Rechtsprechungsänderung in der Schweiz (civil law) und den Vereinigten Staaten (common law), Diss. St. Gallen, Basel 1993, S. 602 f.

60 BGE 120 II 423 E. 7a: «nicht veröffentlichtes Urteil vom 28. April 1992 i.S. H.»

61 Flühmann/Sutter (Fn. 45), S. 1038.

62 Art. 3 Abs. 1 lit. a Ziff. 1, 2 und 5 BGÖ.

63 Botschaft zum Bundesgesetz über die Öffentlichkeit der Verwaltung vom 12. Februar 2003 (BB1 2003 1963), S. 1989.

64 Rainer J. Schweizer/Nina Widmer, in: Stephan C. Brunner / Luzius Mader, Öffentlichkeitsgesetz, Stämpflis Handkommentar, Bern 2008, Art. 3 N 12.
65 Vgl. die Angaben zu den einzelnen Kantonen unter www.oeffentlichkeitsgesetz.ch/deutsch/dasgesetz.

66 Stefanie Hablützel, Graubünden findet seinen Willen zur Transparenz, oeffentlichkeitsgesetz.ch vom 15. Juni 2014: «Seit über zehn Jahren diskutiert das Bündner Parlament über das Öffentlichkeitsprinzip mit dem immer gleichen Ergebnis: Das brauchen wir nicht. Neue Fakten gibt es nicht. Trotzdem stellt sich nun still und leise auch die bürgerliche Mehrheit hinter das Anliegen.»

67 Daniel Glur, CVP will (noch) keine transparente Verwaltung, Zentral+ vom 7. Oktober 2014.

68 Im Kanton Aargau gilt das Gesetz über die Information der Öffentlichkeit, den Datenschutz und das Archivwesen (IDAG) gemäss $§ 2$ Abs. 1 für die 
SZ, SO, TI, UR, VD, ZH) auch für den Zugang zu Dokumenten der Justiz gelten ${ }^{69}$. In den Kantonen mit Öffentlichkeitsgesetz und ohne Ausnahmebestimmung für die Justiz besteht grundsätzlich ein allgemeiner (Ausnahme: AR70) Anspruch auf
Einsichtnahme und Zustellung von Urteilen ${ }^{71}$.

29 Auch wenn sich aus den Informationsund Öffentlichkeitsgesetzen kein Anspruch auf Publikation aller Urteile richterlichen Behörden nur, soweit sie Verwaltungsaufgaben erfüllen. Ebenso im Kanton Schwyz gilt das Gesetz über das Öffentlichkeitsprinzip der kantonalen Verwaltung (Öffentlichkeitsgesetz, OeG) gemäss Art. 2 Abs. 2 lit. c für richterliche Behörden nur, soweit sie Verwaltungsaufgaben erfüllen. Im Kanton Solothurn gilt der Teil betreffend Zugang zu amtlichen Dokumenten des Informations- und Datenschutzgesetzes (InfoDG) gemäss $§ 2$ Abs. 2 lit. a für die Justizbehörden nur, soweit sie Verwaltungsaufgaben erfüllen. Auch im Kanton Tessin ist das Legge sull'informazione e sulla trasparenza dello Stato (LIT) gemäss Art. 2 lit. c nur anwendbar «alle autorità giudiziarie, limitatamente all'informazione attiva e alle loro attività amministrative e di vigilanza». Im Kanton Uri gilt das Gesetz über das Öffentlichkeitsprinzip der kantonalen Verwaltung (Öffentlichkeitsgesetz, OeG) gemäss Art. 2 Abs. 2 lit. c für richterliche Behörden nur, soweit sie Verwaltungsaufgaben erfüllen. Im Kanton Waadt ist das Loi sur l'information (LInfo) gemäss Art. 2 Abs. 1 lit. c nur anwendbar «à l'Ordre judiciaire et à son administration, à l'exclusion de ses fonctions juridictionnelles». Schliesslich gilt auch im Kanton Zürich das Gesetz über die Information und den Datenschutz (IDG) gemäss $§ 2$ Abs. 1 für Gerichte nur, soweit sie Verwaltungsaufgaben erfüllen.

69 Im Kanton Appenzell Ausserrhoden gilt das Gesetz über Information und Akteneinsicht (Informationsgesetz) gemäss Art. 1 Abs. 2 u.a. für alle Behörden und Organe des Kantons. Im Kanton BaselLandschaft wird das am 1. Januar 2013 in Kraft tretende Gesetz über die Information und den Datenschutz (Informations- und Datenschutzgesetz) gemäss $\S 2$ für alle öffentlichen Organe (Abs. 1), nicht aber in hängigen Verfahren der Zivil- und Strafrechtspflege sowie der Verfassungs- und Verwaltungsgerichtsbarkeit (Abs. 2 lit. b und c) gelten. Im Kanton Basel-Stadt gilt das Gesetz über die Information und den Datenschutz (Informations- und Datenschutzgesetz, IDG) gemäss $\S 2$ für alle öffentlichen Organe (Abs. 1), nicht aber in hängigen Verfahren der Zivil- und Strafrechtspflege sowie der Verfassungs- und Verwaltungsgerichtsbarkeit (Abs. 2 lit. b und c). Im Kanton Bern gilt das Gesetz über die Information der Bevölkerung (Informationsgesetz, IG) gemäss Art. 2 Abs. 1 für alle Behörden des Kantons und der Gemeinden. In Art. 27 wird für nicht rechtskräftig abgeschlossene Verwaltungsund Justizverfahren auf die entsprechenden Verfahrensbestimmungen verwiesen. Im Kanton Freiburg gilt das Gesetz über die Information und den $\mathrm{Zu}$ gang zu Dokumenten (InfoG) gemäss Art. 2 lit. a u.a. für die Organe des Staates. Das in Art. 20 gewährte Zugangsrecht gilt allerdings gemäss Art. 21 Abs. 1 lit. a nicht für die Einsichtnahme in Dokumente, die sich auf Zivil-, Straf-, Verwaltungsjustiz- und Schiedsverfahren beziehen. Im Kanton Genf gilt das Loi sur l'information du public, l'accès aux documents et la protection des données personelles (LIPAD) gemäss Art. 3 Abs. 1 lit. a für «les pouvoirs exécutif, législatif et judiciaire cantonaux». Im Kanton Jura ist das Loi sur l'information et l'accès aux documents officiels gemäss Art. 3 Abs. 1 lit. b auch anwendbar «aux autorités judiciaires». Im Kanton Neuenburg ist das Loi sur la transparence des activités étatiques (LTAE) gemäss Art. 2 Abs. 1 anwendbar «aux autorités cantonales et communales», worunter gemäss Art. 2 Abs. 2 lit. c explizit auch «le pouvoir judiciaire» fällt. Im Kanton Schaffhausen ist das Öffentlichkeitsprinzip für alle Behörden in Art. 47 der Kantonsverfassung verankert. Im Kanton St. Gallen ist das Öffentlichkeitsprinzip für alle Behörden in Art. 60 der Kantonsverfassung festgehalten (gemäss Abs. 2 regelt das Gesetz den Zugang zu amtlichen Informationen, dieses Gesetz existiert aber bis heute nicht). Im Kanton Wallis gilt das Gesetz über die Information der Öffentlichkeit, den Datenschutz und die Archivierung (GIDA) gemäss Art. 2 Abs. 1 i.V.m. Art. 3 Abs. 1 lit. a u.a. für die gesetzgebenden, vollziehenden und richterlichen Gewalten des Kantons. Für den Zugang zu amtlichen Dokumenten im Zusammenhang mit hängigen Gerichts-, Verwaltungs- und Schiedsverfahren wird in Art. 12 Abs. 2 auf die Spezialgesetze und Prozessordnungen verweiesen.

70 Gemäss Art. 9 des ausserrhodischen Informationsgesetzes ist (nebst dem Fehlen eines entgegenstehenden überwiegenden öffentlichen oder privaten Interesses) für die Einsicht in amtliche Akten ein berechtigtes Interesse nachzuweisen.

$71 \S 23$ IDG BL; $\S 25$ IDG BS; Art. 27 IG BE; Art. 20 InfoG FR; Art. 24 LIPAD GE; Art. 1 LIADO JU; Art. 20 LTAE NE; Art. 47 Abs. 3 KV SH; Art. 60 KV SG; Art. 12 GIDA VS. 
ergibt, besteht im Internetzeitalter die einfachste Möglichkeit zur Umsetzung des Öffentlichkeitsprinzips in der $\mathrm{Zu}$ gänglichmachung der entsprechenden Informationen über das Internet. Zur passiven, rein duldenden Dimension des Öffentlichkeitsprinzips ist auch für Gerichte eine aktive, kommunikative Dimension hinzugetreten ${ }^{72}$. Dass ein zunehmendes Interesse am Zugang zu diesen Informationen besteht, zeigt ein Blick auf die Opendata- und Openscience-Bewegungen, welche die zeitnahe, vollständige Offenlegung relevanter Primärquellen und den einfachen Zugang auf direkt maschinenlesbare Daten fordern73.Einzelne Kantone haben zudem die grundsätzliche Kostenlosigkeit des Zugangs zu amtlichen Dokumenten gesetzlich verankert74. Wenn für den Aufwand, welche die Zugangsgewährung mit sich bringt, keine Gebühren verlangt werden dürfen, könnten schliesslich auch Kostengründe für (und nicht gegen) die Aufschaltung der Urteile im Internet sprechen.

\section{Pflicht zur Zugänglichmachung ge- mäss ZPO}

3o Unter der Marginalie «Öffentlichkeit des Verfahrens» hält Art. 54 Abs. 1 Satz 2 ZPO fest: «Die Entscheide werden der Öffentlichkeit zugänglich gemacht.» Aus dem Gesetzeswortlaut geht nicht eindeutig hervor, ob eine eigentliche Publikation verlangt ist. Der Begriff des Zugänglichmachens erscheint jedoch nicht erfüllt, wenn lediglich eine Einsichtsmöglichkeit am Sitz des Gerichts angeboten wird. Aus dem Wortlaut ergibt sich ferner, dass weder eine Auswahl von Urteilen noch eine zeitliche Beschränkung des Zugangs zulässig sind.

31 Die Botschaft hält zu diesem Satz lediglich fest, dass die Zugänglichmachung der Entscheide in der Vernehmlassung ausdrücklich gewünscht worden sei75. Aus der Zusammenstellung der Vernehmlassungen zum Vorentwurf ZPO geht hervor, dass eine mit «DDG ZH» abgekürzte Organisation 76 folgenden Wortlaut vorgeschlagen hat77: "Die Verhandlungen sind öffentlich. Die Entscheide werden veröffentlicht oder der Öffentlichkeit zugänglich gemacht.» Zudem hat die Grüne Partei festgehalten 78 : "Aufgrund der in den letzten Jahren aufgetretenen Kontroversen über den Umfang und die Form, in bzw. mit der Gerichtsurteile der Öffentlichkeit (insbes. den Medienschaffenden) zugänglich gemacht werden sollen, halten wir es ferner für sinnvoll, diese Fragen nicht dem kantonalen Recht zu überlassen, sondern in der ZPO-CH entsprechende 'minimal standards' festzulegen.»
72 Wiprächtiger(Fn. 1), S. 146.

$73 \mathrm{Vgl}$. das Manifest «Open Government Data für die Schweiz» des Vereins opendata.ch vom 3. Mai 2011, abrufbar unter http://opendata.ch/manifest/ sowie das Mission Statement der deutschsprachigen Open Science AG, Version 1.0, Oktober 2014.

74 Art. 24 Abs. 1 InfoG FR; Art. 28 Abs. 7 LIPAD GE; Art. 31 LTAE NE; Art. 11 Abs. 1 LInfo VD.
75 Botschaft vom 28. Juni 2006 zur Schweizerischen Zivilprozessordnung (BB1 2006 7221), S. 7275.

76 Gemeint ist wahrscheinlich der Gesetzgebungsdienst des Kantons Zürich (GGD ZH).

77 Zusammenstellung der Vernehmlassungen zum Vorentwurf ZPO, S. 177.

78 Zusammenstellung der Vernehmlassungen zum Vorentwurf ZPO, S. 610. 
In der Lehre wird die Zugänglichmachung gemäss Art. 54 Abs. 1 Satz 2 ZPO als Publikation bzw. Veröffentlichung79 verstanden, wobei eine Publikation im Internet im Vordergrund stehe ${ }^{80}$. Nach Ansicht von Sutter-Somm/Seiler ergibt sich aus dem Sinn und Zweck der Bestimmung, dass lediglich die vollständig ausgefertigten, d.h. mit schriftlicher Begründung versehenen Entscheide der Öffentlichkeit zugänglich zu machen seien ${ }^{81}$. Dieses Argument überzeugt nur beschränkt, weil Sinn und Zweck der Urteilsöffentlichkeit nebst Transparenz der richterlichen Erkenntnisse eben auch die Unterrichtung der Öffentlichkeit über die Rechtspraxis und die Unterbindung von Geheimjustiz sind. Daher müssen m.E. auch Urteile ohne schriftliche Begründung von dieser Bestimmung miterfasst sein.

âhnliche Formulierung wie in Art. 54 ZPO hat der Ständerat bei der Beratung des BGG eingefügt, indem er der bundesrätlichen Fassung des heutigen Art. 27 BGG einen neuen Absatz angefügt hat mit dem Wortlaut: «Die Entscheide sind grundsätzlich in anonymisierter Form der Öffentlichkeit zugänglich zu machen.»82 Der Nationalrat hat den Absatz kommentarlos umformuliert zum später nicht mehr veränderten Wortlaut: «Die Veröffentlichung der Entscheide hat grundsätzlich in anonymisierter Form zu erfolgen.»83 Dies legt den Schluss nahe, dass der Gesetzgeber keinen Unterschied zwischen Zugänglichmachung und Veröffentlichung erkannte. Demgegenüber unterscheidet die zürcherische Kantonsverfassung die beiden Begriffe in Art. $78 \mathrm{KV}$ $\mathrm{ZH}$, indem Abs. 1 festhält, dass Rechtspflegeentscheide auf angemessene Weise der Öffentlichkeit zugänglich gemacht werden, während Abs. 2 besagt, dass die Entscheidungspraxis veröffentlicht wird. Auch die Zugänglichmachung nach Abs. 1 verlangt jedoch eine aktive Information über die getroffenen Entscheide, d.h. sie wird mit einem passiven Dulden richterlicher Behörden gegenüber privater Einblicknahme in die justizielle Tätigkeit allein nicht erfüllt ${ }^{84}$.

34 Auch wenn die in Art. 54 Abs. 1 Satz 2 ZPO verlangte Zugänglichmachung nicht zwingend als Pflicht zur Publikation verstanden werden muss, so ist dies doch die naheliegendste Lesart. Ganz abgesehen davon, dass eine Veröffentlichung der Entscheide im Internet die einfachste, kostengünstigste und auch publikumsfreundlichste Möglichkeit des Zugänglichmachens darstellt.

\section{Umfang der Publikation}

35 Da in der Schweiz keine Bindung an die Rechtsprechung des höchsten Gerichts besteht ${ }^{85}$, ist bei der Publikationswürdigkeit schon aus diesem Grund eine Unterscheidung nach Instanzen abzulehnen.
79 Franz Schenker, in: Baker \& McKenzie, Schweizerische Zivilprozessordnung (ZPO), Stämpflis Handkommentar, Bern 2010, Art. 54 N 5; Thomas Sutter-Somm /Benedikt Seiler, in: Thomas SutterSomm / Franz Hasenböhler / Christoph Leuenberger (Hrsg.), Kommentar zur Schweizerischen Zivilprozessordnung, Art. 54 N 13; Jörg Paul Müller / Markus Schefer, Grundrechte in der Schweiz, Bern 2008, S. 977.
80 Sutter-Somm/Seiler (Fn. 79), Art. 54 N 13.

81 Sutter-Somm/Seiler (Fn. 79), Art. 54 N 14.

82 AB 2003 S 894.

83 AB 2004 N 1588.

84 Vogel (Fn. 48), Art. 78 N 29.

85 Hans Peter Walter, Interne richterliche Unabhängigkeit, in: «Justice - Justiz - Giustizia» 2005/1, Rz. 21. 
Dass erst- und zweitinstanzliche Urteile regelmässig von genauso grossem Interesse sind wie diejenigen des Bundesgerichts, zeigen schon der Abdruck und die Besprechung solcher Richtersprüche in der juristischen Literatur. Gerade in Rechtsgebieten, die sich rasch weiterentwickeln, kann zudem eine juristische Frage innerhalb des Zeitrahmens, den der Instanzenzug konsumiert, bereits wieder veraltet sein. Schliesslich sei die These gewagt, dass gut begründete Urteile die Parteien überzeugen und sie deshalb tendenziell von einem Weiterzug absehen lassen. Auch dies führt zu einem mindestens so grossen öffentlichen Interesse an erstund zweitinstanzlichen Urteilen wie an Bundesgerichtsentscheiden.

Die Tatsache, dass erst- und zweitinstanzliche Entscheide seltener zitiert werden als diejenigen des Bundesgerichts, ist die Folge (und nicht etwa ein Grund für die Beibehaltung) der mangelnden Zugänglichkeit dieser Richtersprüche. Eine Unterscheidung von massgebendem und nicht massgebendem Fallrecht je nach Instanz, d.h. die stärkere (oder gar ausschliessliche) Berücksichtigung von höchstinstanzlichen Urteilen bei der Publikation ist somit abzulehnen.

Weiter ist zu prüfen, ob eine Auswahl von publikationswürdigen Urteilen aus der Gesamtheit der Entscheide einer bestimmten Instanz angezeigt ist. Das Bundesgericht hat während 125 Jahren ausschliesslich Urteile in Form der BGE, d.h. der amtlichen Sammlung ausgewählter Entscheide bzw. der wichtigsten Erwägungen daraus, publiziert. Dies war nur schon deshalb der einzig gangbar Weg, weil eine Publikation zu dieser Zeit ausschliesslich in gedruckter Form möglich war und der Druck sämtlicher Entscheide des Bundesgerichts hohe Kosten und Papierverbrauch mit sich gebracht hätte. Hinzu kommt aber auch die fehlende Möglichkeit zur effizienten Erfassung der Relevanz eines Urteils für eine bestimmte Frage. Die einzige Hilfe bestand in einer groben Einteilung nach Rechtsgebieten und kurzen Zusammenfassung in Form von Regesten.

38 Demgegenüber beginnt heute eine Recherche nach relevantem Fallrecht wohl fast immer mit einer computergestützten Suche; lediglich für die Lektüre der gefundenen Urteile wird allenfalls auf eine gedruckte Fassung zurückgegriffen. Eine Internetpublikation vereinigt die Vorteile der allgemeinen Verfügbarkeit und der tiefen Kosten sowohl auf Seiten des Gerichts als auch des Rechtsuchenden. Für eine Auswahl von zu verföffentlichenden Urteilen spricht die Tatsache, dass viele Entscheide das Ergebnis reiner Routinearbeit sind oder einen derart spezifischen Einzelfall betreffen, dass sie fast nur für die konkret betroffenen Parteien von Interesse sein können. Dies dürfte unbestritten sein; das Problem der Auswahl ist jedoch ein anderes und zwar die Möglichkeit zur Nichtpublikation aus sachfremden Gründen.

39 Auch wenn eine nachvollziehbare und hilfreiche Vorauswahl getroffen wird, bleibt immer der Verdacht, dass sich unter den nicht publizierten Entscheiden der eine oder andere juristisch oder anderweitig interessante Fall befinden könnte. Mögliche sachfremde Gründe für eine unterlassene Publikation können in einer wenig geglückten Begründung oder einem Abwarten, ob die eingeschlagene Praxis in 
späteren Urteilen ihre Probe besteht, liegen ${ }^{86}$. Auch das Bundesgericht hat die Erfahrung gemacht, dass die «wohlgemeinte Dienstleistung des Bundesgerichts in Form einer Ausscheidung der gänzlich unbedeutenden Urteile [...] nicht mehr gefragt [ist]; Vertrauen in eine gute Auswahl durch das Bundesgericht hat in den Augen Dritter nicht mehr den früheren Stellenwert.» 87

Eine Analyse der Publikationspraxis des Bundesgerichts hat allerdings gezeigt, dass sogar Praxisänderungen teilweise unpubliziert geblieben sind ${ }^{88}$. Eine selektive Publikation ist systemimmanent dazu verurteilt, sich selbst Lügen strafen zu müssen, weil im Nachhinein unveröffentlichte Urteile eben doch als Präjudizien herbeigezogen werden ${ }^{89}$. Das Bundesgericht hat die Zeichen der Zeit erkannt und ist dazu übergegangen, sämtliche Endund Teilentscheide sowie die vom Abteilungspräsidium bezeichneten Vor- und Zwischenentscheide im Internet aufzuschalten ${ }^{90}$. Dies ist m.E. der einzig richtige Weg. Eine inhaltliche Auswahl von zu publizierenden Urteilen durch das Gericht ist primär aus Transparenzgründen, aber auch wegen der fehlenden Vorhersehbarkeit des Präjudizcharakters eines Entscheids abzulehnen ${ }^{91}$.

\section{Argumente gegen eine umfassende Publikationspflicht}

\section{Informationsüberflutung}

41 Als das Bundesgericht vor Jahren den Medien die Versorgung mit sämtlichen Urteilen angeboten hat, haben diese offenbar entrüstet abgelehnt, mit der Begründung, dies käme infolge der zu erwartenden Informationsüberflutung einer indirekten Informationsverweigerung gleich92. Dieses Argument mag Gültigkeit gehabt haben, solange man noch keine computergestützten Recherchemöglichkeiten, insbesondere die Volltextsuche, zur Verfügung hatte93.

42 Heute ist jedoch die umfassende Kenntnis des massgebenden Fallrechts ohnehin nur noch in sehr begrenzten Rechtsgebieten möglich. Weit wichtiger ist die Kompetenz zum Auffinden der relevanten Informationen anhand von Suchfunktionen, was sich schon daran zeigt, dass juristische Recherchekurse auf unterschiedlichen Niveaus angeboten werden 94 . Dass es einfacher ist, alle relevanten Urteile zu kennen, wenn die Zahl derselben tief bleibt, liegt auf der Hand. Dies darf jedoch nicht dazu führen, dass Urteile mit dieser Begründung nicht oder nur zurückhaltend veröffentlicht werden.

\footnotetext{
Bosshardt (Fn. 2), S. 347.

Tschümperlin (Fn. 17), N 11.

Probst (Fn. 59), S. 605.

Probst (Fn. 59), S. 620.

Tschümperlin (Fn. 17), N 8.

91 Vgl. auch Vogel (Fn. 48), Art. 78 N 33, wonach zwar nicht sämtliche Urteile publiziert werden müssten, aufgrund der schwierigen Einteilung in Leitentscheide und andere Urteile aber nebst der offiziellen Sammlung eine Datenbank mit weiteren
}

Urteilen einzurichten sei. Nach der vorliegend verwendeten Terminologie ist die Aufnahme eines Urteils in eine Online-Datenbank auch als Publikation $\mathrm{zu}$ verstehen.

92 Tschümperlin (Fn. 17), N 8.

93 So auch Vogel (Fn. 48), Art. 78 Fn. 64.

94 Als Beispiel sei auf die im Rahmen des Studiums an der Universität Bern durchgeführten Kurse verwiesen. 


\section{Anonymisierungsaufwand}

43 Ein weiteres, oft gegen die Publikation von Gerichtsentscheidungen vorgebrachtes Argument, ist die Anonymisierung bzw. der dabei entstehende Aufwand. In diesem Sinne soll nachfolgend zunächst ein Blick auf die tatsächliche Höhe dieses Aufwands geworfen werden, bevor anschliessend die Notwendigkeit der ausnahmslosen Anonymisierung sämtlicher Urteile hinterfragt wird.

\subsection{Aufwand der Anonymisie- rung}

44 In der in allen Kantonen durchgeführten Umfrage zur Urteilspublikation wurde auch die Frage gestellt, wie gross schätzungsweise der Anonymisierungsaufwand pro Urteil ist. Die von 17 Kantonen gemachten Angaben variieren zwischen 5 und 60 Minuten (Ausnahme Kanton Wallis: 120 Minuten), der Durchschnitt aller gegebenen Antworten beträgt 33 Minuten.

45 Der Kanton Zürich beschäftigt ein Anonymisierungsteam mit 100 Stellenprozenten, das sich überwiegend aus RechtsstudentInnen zusammensetzt95. Diese können von zuhause aus arbeiten und anonymisieren rund 200 Entscheide pro Monat.
Ab Zustellung eines Urteils an die Parteien bis zur anonymisierten Online-Publikation vergehen durchschnittlich zweieinhalb Monate.

46 Der Anonymisierungsaufwand hängt auch massgeblich davon ab, wie anonymisierungsfreundlich ein Urteil verfasst wird ${ }^{96}$. Ist von Beginn an klar, dass ein Entscheid später publiziert werden soll, kann z.B. darauf geachtet werden, dass die Parteinamen nur im Rubrum und im Dispositiv vorkommen, während im Sachverhalt und den Erwägungen die Bezeichnungen gemäss der Rollenverteilung im Prozess (Beschwerdeführer / Gesuchsteller / Beschwerde- / Gesuchsgegner) verwendet werden. Schliesslich kann die Anonymisierung auch durch den Einsatz von Spezialsoftware 97 zumindest teilweise automatisiert und der Aufwand dadurch reduziert werden.

\subsection{Notwendigkeit der Anonymi- sierung}

47 Die Anonymisierung von zu publizierenden Urteilen wird im Bundesgerichtsgesetz, im Verwaltungsgerichtsgesetz, im Strafbehördenorganisationsgesetz und in verschiedenen kantonalen Gesetzen oder Richtlinien standardmässig verlangt ${ }^{98}$. In

werden.»

97 Als Beispiel sei auf die Weblaw Anonymisierung verwiesen, vgl. www.weblaw.ch > Competence > Technology > Gerichtslösungen.

98 Art. 27 Abs. 2 BGG, Art. 29 Abs. 2 VGG, Art. 63 Abs. 2 StBOG; Ziff. IV.3. des Kreisschreibens des Obergerichts des Kantons Aargau über die Akteneinsicht, die Herausgabe von Akten und die Information über Gerichtsverfahren; Art. 10 Abs. 4 des bernischen Informationsreglements der Zivil-, Straf- und Jugendgerichtsbehörden; Art. 20 Abs. 4 des Loi sur l'information du public, l'accès aux documents et la protection des données personnelle des Kantons Genf; § 123 der luzernischen Verord- 
den Entwürfen des Bundesgerichtsgesetzes, des Verwaltungsgerichtsgesetzes, und des Strafgerichtsgesetzes war die Anonymisierung allerdings noch nicht vorgesehen 99 , d.h. sie wurde erst im Rahmen der parlamentarischen Beratung eingefügt.

Nichtsdestotrotz ist die Rechtslage für Entscheide des Bundes-, des Bundesverwaltungs- und des Bundesstrafgerichts damit eindeutig. Es ist jedoch fraglich, ob eine durchgehende Anonymisierung sinnvoll und de lege ferenda geboten ist ${ }^{100}$. Art. 25 PatGG enthält denn auch keine solche Vorgabe mehr und das Bundespatentgericht hat in seinem Informationsreglement die Anonymisierung explizit als Ausnahme und damit die Nichtanonymisierung als Regel statuiert. Art. 3 Abs. 3 IR-PatGer lautet: «Die Veröffentlichung erfolgt in nicht anonymisierter Form, es sei denn, der Schutz privater oder öffentlicher Interessen erfordert eine Anonymisierung. Diese kann von Amtes wegen vorgenommen werden. Bei privaten Inte- ressen wird die Anonymisierung vorgenommen, wenn sie beantragt wird und begründet erscheint.» Der zweite Satz («Diese kann von Amtes wegen vorgenommen werden.») wurde erst per 1. April 2013 ergänzt. In der Zeit ab Inkrafttreten des Reglements am 1. Januar 2012 bis zur Ergänzung des zweiten Satzes per 1. April 2013 gab es somit keine gesetzliche Grundlage zur Anonymisierung von Amtes wegen ${ }^{101}$.

49 Schutzzweck der Urteilsöffentlichkeit ist u.a. die Schaffung von Transparenz. Darunter fällt auch die Möglichkeit der Öffentlichkeit zur Kontrolle der Gleichbehandlung durch die Gerichte ${ }^{102}$. Dass einflussreiche Personen von der Justiz nicht anders als alle anderen behandelt werden, kann nur nachgewiesen werden, wenn die Urteile nicht anonym sind ${ }^{103}$. Wenn zum Vornherein bekannt ist, dass eine berühmte Persönlichkeit vor Gericht steht, wird diese Überwachungsfunktion i.d.R. durch die Medien wahrgenommen. Dies dürfte jedoch nur selten der Fall sein ${ }^{104}$, nung zum Gesetz über die Organisation der Gerichte und Behörden in Zivil- und Strafverfahren; Art. 35 der st.gallischen Gerichtsordnung. § 3 Abs. 3 der zürcherischen Verordnung der obersten kantonalen Gerichte über die Information über Gerichtsverfahren und die Akteneinsicht bei Gerichten durch Dritte sieht für die Akteneinsicht eine Einzelfallentscheidung vor.

99 Art. 25 Entwurf BGG (BB1 2001 4480), Art. 26 Entwurf VGG (BB1 2001 4539), Art. 24 Entwurf SGG (BB1 2001 4517).

100 Saxer (Fn. 96), S. 423, bezeichnet die Anonymisierung für die Öffentlichkeit, die Rechtssuchenden und deren Rechtsvertreter als «leidiges Thema»; vgl. auch Markus Felber, Der Journalist als Mittler und Wächter, in: Marianne Heer/Adrian Urwyler (Hrsg.), Justiz und Öffentlichkeit - Justice et public, Bern 2007, S. 125 ff., S. 130; Vogel (Fn. 48), Art. 78 N 21.

101 Art. 3 Abs. 3 IR-PatGer (Stand am 1. Januar 2012): «Die Veröffentlichung erfolgt in nicht anonymisier- ter Form, es sei denn, der Schutz privater oder öffentlicher Interessen erfordert eine Anonymisierung. Bei privaten Interessen wird die Anonymisierung vorgenommen, wenn sie beantragt wird und begründet erscheint.»

102 BGE 137 I 16 E. 2.2 S. 19: «Das Prinzip der Justizöffentlichkeit und die daraus abgeleiteten Informationsrechte sind von zentraler rechtsstaatlicher und demokratischer Bedeutung. Sie sorgen für Transparenz in der Rechtspflege, was eine demokratische Kontrolle durch das Volk erst ermöglicht, [...]».

103 Raselli (Fn. 9), S. 35.

104 Erstens bestehen in vielen Kantonen auch für Medien nur eingeschränkte Möglichkeiten, um zu erfahren, welche Personen wann und wo vor Gericht stehen. Zweitens führt der Spardruck auch in Medien wie der NZZ dazu, dass die Gerichtsberichterstattung an Gewicht verliert. Siehe dazu Claudia Schoch, Ein kritischer Beobachter und Mahner, nzz.ch vom 29. Juni 2013 sowie René Zeller, Rollenwechsel von Claudia Schoch, nzz.ch vom 30. 
sodass in aller Regel nach einer öffentlichen Verkündung ohne Teilnahme der Öffentlichkeit inkl. Medien keine Möglichkeit mehr besteht, um herauszufinden, ob wirklich alle von einem Gerichtsurteil Betroffenen unabhängig von ihrer Herkunft und ihrem Einfluss gleich behandelt werden. Die demokratische Öffentlichkeit hat ein Interesse daran, gerichtliche Entscheidungen vollständig kennenzulernen, d.h. mitsamt Namen der Beteiligten $^{105}$. Dies bestätigt auch das Bundesgericht, wenn es festhält: «Sofern keine besonderen, schutzwürdigen Geheimhaltungsinteressen - bei deren Vorliegen allenfalls die Öffentlichkeit ausnahmsweise von den Verhandlungen ausgeschlossen werden könnte - ersichtlich sind, hat der Berechtigte Anspruch auf Kenntnisnahme des vollständigen, ungekürzten und nicht anonymisierten Urteils»106.

Damit soll nicht einer generellen Abkehr von der Anonymisierung von Gerichtsentscheiden das Wort geredet werden. Es gibt zahlreiche Fälle, gerade im Straf-, im Sozialversicherungs- oder im Familienrecht, in denen eine Anonymisierung mit Blick auf die Persönlichkeitsrechte der Betroffenen wichtig erscheint. In vielen anderen Rechtsgebieten, so z.B. im Wirtschafts- (inkl. Steuer-) oder im Verwaltungsrecht ${ }^{107}$, ist jedoch auf den ersten Blick kein Grund zur generellen Anonymisierung ersichtlich. Auch das Bundesgericht hat festgehalten, dass mit der Anonymisierung eines Urteils einem berechtigten Interesse der Beschwerdeführerin am Persönlichkeits- und Datenschutz Rechnung getragen wird ${ }^{108}$. Ob ein in diesem Sinne berechtigtes Interesse vorliegt, wird indessen nicht im Einzelfall geprüft, was mit Blick auf Art. 27 Abs. 2 BGG (Veröffentlichung grundsätzlich in anonymisierter Form) auch nichts notwendig erscheint. Die Frage nach der Verfassungsmässigkeit von Art. 27 Abs. 2 BGG ist im Rahmen des vorliegenden Beitrags mit Blick auf Art. 190 BV obsolet.

51 Gemäss Bundesrichter Seiler wird von einer Anonymisierung beim Bundesgericht insbesondere dann abgesehen, wenn eine öffentliche Verhandlung oder Beratung stattfindet, weil dann ohnehin die Namen der Parteien bekannt sind ${ }^{109}$. Dies würde allerdings dafür sprechen, grundsätzlich alle Urteile unanonymisiert zu publizieren, weil die Namen der Parteien mit wenigen Ausnahmen (Opferhilfe, Steuerrecht, internationale Rechtshilfe) in den Dispositiven vor Ort eingesehen werden

\footnotetext{
September 2014 sowie Christian Müller, NZZ verliert juristisches Gewissen, infosperber.ch vom 1. Oktober 2014: «Eine andere Stimme im Inland-Ressort der NZZ, die unser Land differenzierter zu verstehen und zu erklären versuchte, als ihr direkter Chef, war Claudia Schoch, von der Ausbildung her Juristin und spezialisiert auf Themen des Staatsund Völkerrechts. Sie wird künftig nur noch Rechtskonsulentin der NZZ sein, aber nicht mehr schreiben. Schade! [...] Der letzte Artikel von Claudia Schoch in der NZZ ist es wert, als Testament gelesen zu werden. Sie macht darauf aufmerksam, dass auch demokratisch gefällte Entscheide internationales Recht, insbesondere die Menschenrechte,
}

nicht einfach beiseite schieben können.»

105 Raselli (Fn. 9), S. 35.

106 BGE 124 IV 234 E. 3e.

107 Saxer (Fn. 96), S. 423.

108 BGE 133 I 106 E. 8.3.

109 Hansjörg Seiler, in: Hansjörg Seiler / Nicolas von Werdt / Andreas Güngerich, Bundesgerichtsgesetz (BGG), Stämpflis Handkommentar, Bern 2007 , Art. 27 N 8: «Dies gilt insbesondere dann, wenn eine öffentliche Verhandlung (Art. 59 BGG) stattfindet, weil dabei ohnehin die Namen der Parteien bekannt sind». Gemäss Rücksprache mit dem Autor gilt dies auch für öffentliche Beratungen. 
können ${ }^{110}$ und damit in vergleichbarem Masse bekannt sind wie bei der Durchführung einer öffentlichen Beratung oder Verhandlung.

Dass der Grundsatz der Anonymisierung auch aus Sicht der Menschenrechte nicht zwingend ist, zeigt zudem ein Blick auf die Praxis des Europäischen Gerichtshofes für Menschenrechte, die i.d.R. anhand der Parteinamen zitiert wird ${ }^{111}$. Die Namen der Beschwerdeführer werden in aller Regel publiziert, obwohl nicht selten Bereiche betroffen sind, die besonders sensibel sind und bei denen eine Nichtanonymisierung in der Schweiz undenkbar wäre, wie z.B. Kindsentführung ${ }^{112}$, Verpflichtung zur Ermöglichung von Suizid ${ }^{113}$, Adoption ${ }^{114}$, Geschlechtsanpassung ${ }^{115}$, die Umstände der Bestattung eines totgeborenen Kindes ${ }^{116}$ oder die Kenntnis der eigenen Abstammung 117. Mit Blick auf die Praxis des EGMR wird denn auch vertreten, dass die Bedeutung der Anonymisierung in der Schweiz überschätzt wird ${ }^{118}$.

53 Auch im Vereinigten Königreich, in Frankreich und in Italien werden die Namen aller Prozessbeteiligten inkl. Parteien veröffentlicht, nicht aber in Österreich und Deutschland ${ }^{119}$. In der Schweiz wurden die Namen der Prozessparteien bis in die 1980er-Jahre in der Amtlichen Sammlung bekanntgegeben. Die Änderung der Veröffentlichungspraxis soll mit dem Inkrafttreten des Datenschutzgesetzes zusammenhängen, auch wenn nicht klar ist, inwiefern in der Publikation von Parteinamen eine Verletzung des Datenschutzes liegen soll120.

\section{Fazit}

54 Urteile vermögen ihre Wirkung im Rechtsstaat nur dann zu entfalten, wenn sie bekannt sind. Eine einzelfallweise $\mathrm{Zu}-$ stellung von schriftlichen Urteilen auf Anfrage genügt zwar vielleicht den Anforderungen der Verfassung, führt aber nur in sehr beschränktem Mass zur geforderten Transparenz. Nur mit einer Publikation sämtlicher Urteile aller Instanzen wird die von der Verfassung verlangte Urteilsöffentlichkeit eindeutig erfüllt, aber auch die Pflicht zur Publikation normativer Akte sowie die Zivilprozessordnung weisen den Weg in diese Richtung.

55 Nachdem das Bundesgericht zunächst nur eine Auswahl und dann Schritt für Schritt immer mehr und schliesslich sämtliche End- und Teilentscheide publiziert hat, ist inzwischen eine ähnliche Tendenz bei den Urteilen der obersten
110 Tschümperlin (Fn. 17), N 13; Art. 59 Abs. 3 BGG.

111 Zitierregeln des Bundesgerichts, Ziff. 2.5 (Urteile des Europäischen Gerichtshofes für Menschenrechte).

112 Urteil Küçük gegen die Türkei und die Schweiz vom 17. Mai 2011 (Nr. 33362/04); Urteil Neulinger und Shuruk gegen die Schweiz vom 6. Juli 2010 (Grosse Kammer) (Nr. 41615/07).

113 Urteil Haas gegen die Schweiz vom 20. Januar 2011 (Nr. 31322/07).

114 Urteil Schwizgebel gegen die Schweiz vom 10. Juni 2010 (Nr. 25762/07).

115 Urteil Schlumpf gegen die Schweiz vom 8. Januar 2009 (Nr. 29002/06).
116 Urteil Hadri-Vionnet gegen die Schweiz vom 14. Februar 2008 (Nr. 55525/00).

117 Urteil Jäggi gegen die Schweiz vom 13. Juli 2006 (Nr. 58757/00).

118 Tschümperlin (Fn. 17), N 25.

119 Kurt Siehr, Veröffentlichung von Gerichtsentscheidungen - Zur Anonymisierung oder Veröffentlichung von Namen der Beteiligten eines Zivilverfahrens, in: Luc Thévenoz / Norbert Reich (Hrsg.), Droit de la consommation / Konsumentenschutzrecht / Consumer law, Liber amicorum Bernd Stauder, Genf 2006, S. 469 ff., S. 470 ff.

120 Siehr (Fn. 119), S. 481. 
kantonalen Gerichte feststellbar. Dies ist zu begrüssen, zumal diese Urteile in ihrer rechtsstaatlichen Funktion denjenigen des Bundesgerichts kaum nachstehen. Die Geschichte hat gezeigt, dass die Publikation einer Auswahl von Urteilen nicht zur geforderten Transparenz führt. Hinzu kommt, dass mit der Digitalisierung und den damit einhergehenden Recherchemöglichkeiten heute keine guten Gründe mehr für eine inhaltliche Vorauswahl des zu Publizierenden bestehen. Es ist somit davon auszugehen, dass auch die obersten kantonalen Gerichte früher oder später dazu übergehen werden, all ihre Urteile zu publizieren. Das Gleiche gilt für die erstinstanzlichen Gerichte, deren Urteile heute in den meisten Kantonen noch gar nicht veröffentlicht werden.

56 Den verfassungsrechtlichen Vorgaben kann in der Regel nur mit einem Gang bis vor Bundesgericht ${ }^{121}$ oder sogar an den Europäischen Gerichtshof für Menschenrechte zum Durchbruch verholfen werden. Es besteht aber eine gewisse Hoffnung, dass Art. 54 ZPO dem Gedanken des Zugänglichmachens über das Zivilrecht hinaus zum Durchbruch verhilft.

121 Markus Felber, Justiz als Nonvaleur?, nzz.ch vom 29. Juni 2013: «Die meisten kantonalen Gerichte tun sich dagegen noch äusserst schwer mit Transparenz und halten ihre Erkenntnisse mit bisweilen abenteuerlicher Begründung, aber im Streitfall ohne Chance auf Erfolg, unter Verschluss.» 